\title{
My_Healthy_Shape Guide: An interactive Educational-Health Tool for Malaysian Based on Body Shape
}

\author{
Hasfaliza TUMIN ${ }^{1}$, Asma AHMAD SHARIFF ${ }^{1,2}$, Amir Feisal MERICAN ${ }^{1,3}$ \\ ${ }^{1}$ Centre of Research for Computational Sciences and Informatics in Biology, Bioindustry, Environment, \\ Agriculture and Healthcare (CRYSTAL), University of Malaya, Malaysia; \\ ${ }^{2}$ Centre of Foundation Studies in Science, University of Malaya, Malaysia; \\ ${ }^{3}$ Institute of Biological Sciences, Faculty of Science, University of Malaya, Malaysia
}

http://dx.doi.org/10.15221/14.275

\begin{abstract}
This study is concerned with developing an interactive educational-health tool, My_Healthy_Shape Guide for Malaysian based on gender, height, weight, body shape and age. In addition to manual anthropometric measurements of height and weight, a three dimensional (3-D) body scanner is used to collect digitized body shape and the data obtained are stored in a specialized human shape database. The scanner captures high quality digital information of the body shape of an individual in just a few minutes. The body shape data as well as the manual anthropometric measurements will be use to study the individual's health status in relation to his/her dietary behaviors and physical activities. This paper presents the conceptual framework of our study, consisting of (1) establishing a web-based 3D human shape database for Malaysian using MySQL (2) formulating mathematical models to generate feasible solutions for predicting healthy diet for an individual based on his/her body shape and (3) developing an interactive educational-health tool, My_Healthy_Shape Guide for Malaysian that would provide healthy daily diet recommendations that promotes good health and lifestyle for an individual.
\end{abstract}

Keywords: 3d body scanning, mathematical programming, educational-health tool

\section{Introduction}

In recent years, the world has seen rapid increase in food prices, which subsequently gives rise to many issues and problem. As described in the report by Malaysian-German Chamber of Commerce and Industry, the food price inflation erodes purchasing power, increasing the possibility of food deprivation and malnutrition [1]. When they buy cheap food, their concerns may be on food that will fulfill their calorie requirements such as food rich in carbohydrate and fat. This will have detrimental effect on their health. Thus, it is important to introduce My_Healthy_Shape Guide that can help the people to consume optimal diet. Optimal diet is a diet that contains several combination of healthy food to be taken daily based on an individual's nutritional requirement and body health. With the food and lifestyle guides, the people can select the type and amount of food that promotes healthy diet and lifestyle. Besides that, females with $58.3 \%$ had a significantly higher mean body dissatisfaction score than males with $58.3 \%$, indicating their preference for a slimmer body shape [2, 3]. As having a distorted body image may lead to negative effects such as unhealthy eating habits and disordered eating behaviors, it is recommended that appropriate educational efforts on body image be incorporated into school health activities for adolescents.

Malaysia is currently working towards achieving the Millennium Development Goals [4]. The Tenth Malaysia Plan (2011-15) addresses several challenges and opportunities, including increasing expectations for quality of health care; the growing pressure on the public health care system; the increasing workload in public hospitals, stretching capacity; shifting lifestyles and demography; and advances in technology. In response to these challenges, the Government of Malaysia is reforming the health care delivery system by transforming delivery of health care; increasing quality, capacity and coverage of the health care infrastructure; shifting towards prevention rather than treatment; and increasing the quality of human resources for health

Selection of diets by quantitative technique is becoming increasingly common because nutritionists are becoming more aware of the dangers of overdoses of some nutrients as well as the need for a balanced intake of nutrients and undertaking appropriate physical activities [5]. This study deals with the theoretical aspects of developing an interactive educational-health tool, My_Healthy_Shape Guide for Malaysian and describes the research that have been taken to accomplish the objectives. It commences with the conceptual framework, which gives an overview of the procedure carried out from the beginning to the end. 


\section{Description of the Conceptual Framework}

This study is concerned with using mathematical programming (MP) in developing an interactive diet planner in recommending daily dietary intake based on age-gender groups, physical activities and health status. MP model is utilized to determine an individual's healthy dietary intake. Figure 1 shows the conceptual framework in designing the theory that attempt to connect to all aspects of inquiry on this study such as data collection, methodology and analysis. It consists of three phases: (1) establishing a web-based 3D human shape database for Malaysian using MySQL (2) formulating mathematical models to generate feasible solutions for predicting healthy diet for an individual based on his/her body shape and (3) establishment of an interactive web-based educational-health system, My_Healthy_Shape Guide for Malaysian.

Phase I involves data collection, data recording, data analysis and development of specialized databases. Conditions were set for age-gender groups, list of food and drinks, nutrient content, physical activities and health status. The data on nutrient contents of food and requirement of nutrients based on age-gender groups were collected from the Ministry of Health (MOH), Malaysia. The nutrient requirement for each age-gender group, nutrient content, physical activities and health status are described as the parameters in development of the system. A database of local food and drinks and age-gender groups are constructed to be used in the model building in determining the optimal diet for each age-gender groups. A database of body measurement is also constructed to be used in model building to determine the health status and recommend physical activities suited for each subjects.

In Phase II, the MP model is designed to check the chosen daily menus with the corresponding serving sizes of food and drink satisfy the age-gender group nutrient requirement and the chosen recommended physical activities satisfy the health status. Then, validation and verification process would take place. These processes are important in mathematical modelling since these process increase the model credibility. Verification ensures that the model satisfies all descriptions, specifications and assumptions whereas validation is the process of determining whether the model represents the real situation to a sufficient level of accuracy.

In Phase III, the interactive educational-health planner and the interface of Web-based system is developed. Web-based system was designed to select the correct combination of food with the correct amount on the user daily menus and level of daily physical activities. Selected findings are highlighted because the solution for this problem may not be unique.

Figure 2 shows the architecture of the My_Healthy_Shape Guide as an intelligent planning system. The user interacts through the user interface within the system. The system consists of two main modules, the management and result generating modules. The management module is designed to manage information relating to user information while the result generating module is designed to interactively assist users in planning suitable menus and physical activities for good health and lifestyle.

In the management module, two types of user inputs are needed. First is the input on personal details, health status and daily activities. Second is on body measurement using the 3D body scanner. If all the user input data are complete, then the system will proceed to another module. Otherwise, the user needs to insert additional input. All the information available in the management module is used in the result generating module. In the result generating module, PHP is used to match all the user inputs with information in the databases before the system generates the recommended dietary intake and physical activities. This database is used for food-analysis and health-analysis purposes. The selected food recommended by the system must satisfy user's nutrient requirements while the recommended physical activities must satisfy user's health status. 


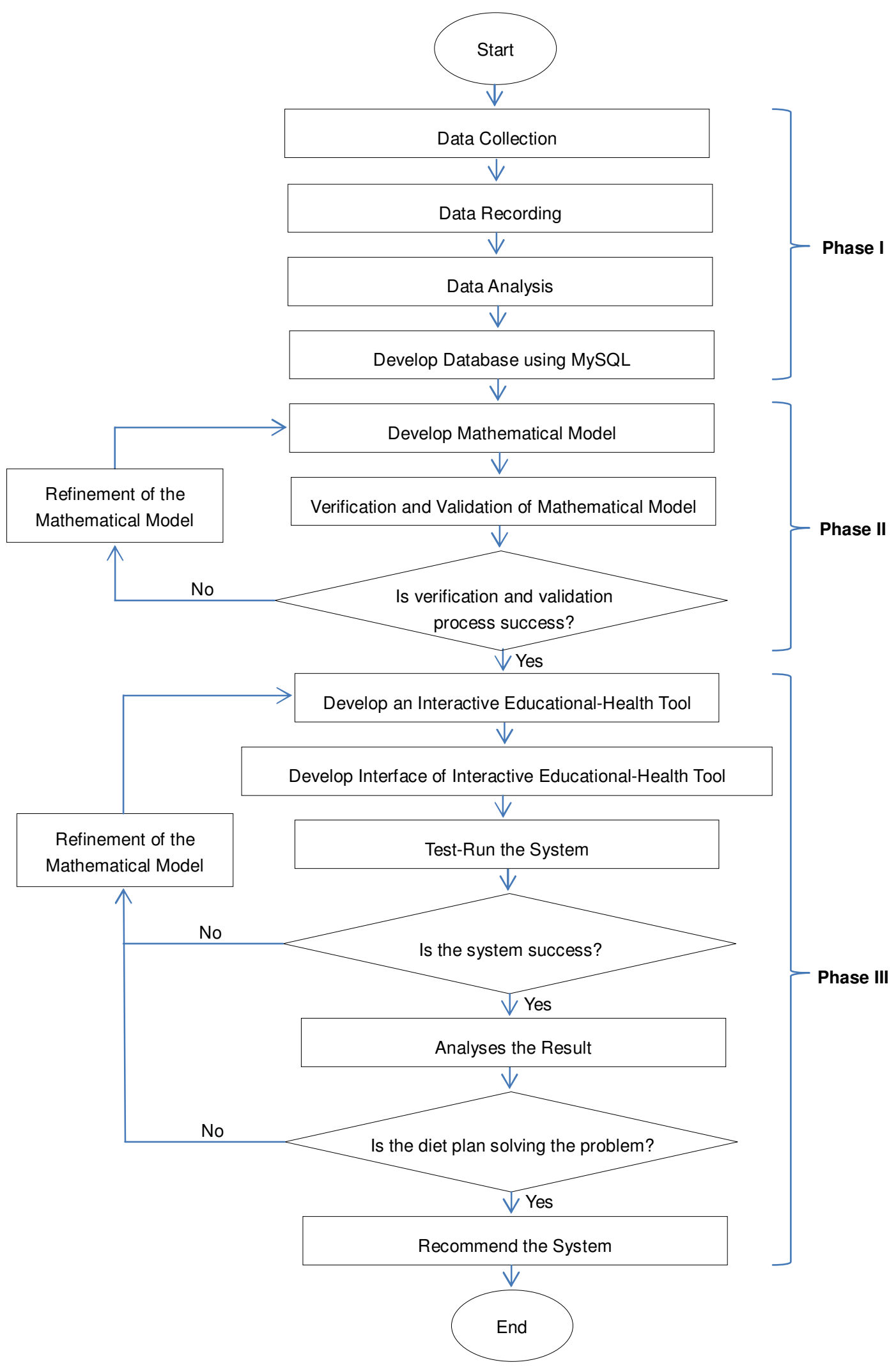

Fig. 1. The Conceptual Framework in Designing the My_Healthy_Shape Guide for Malaysian 


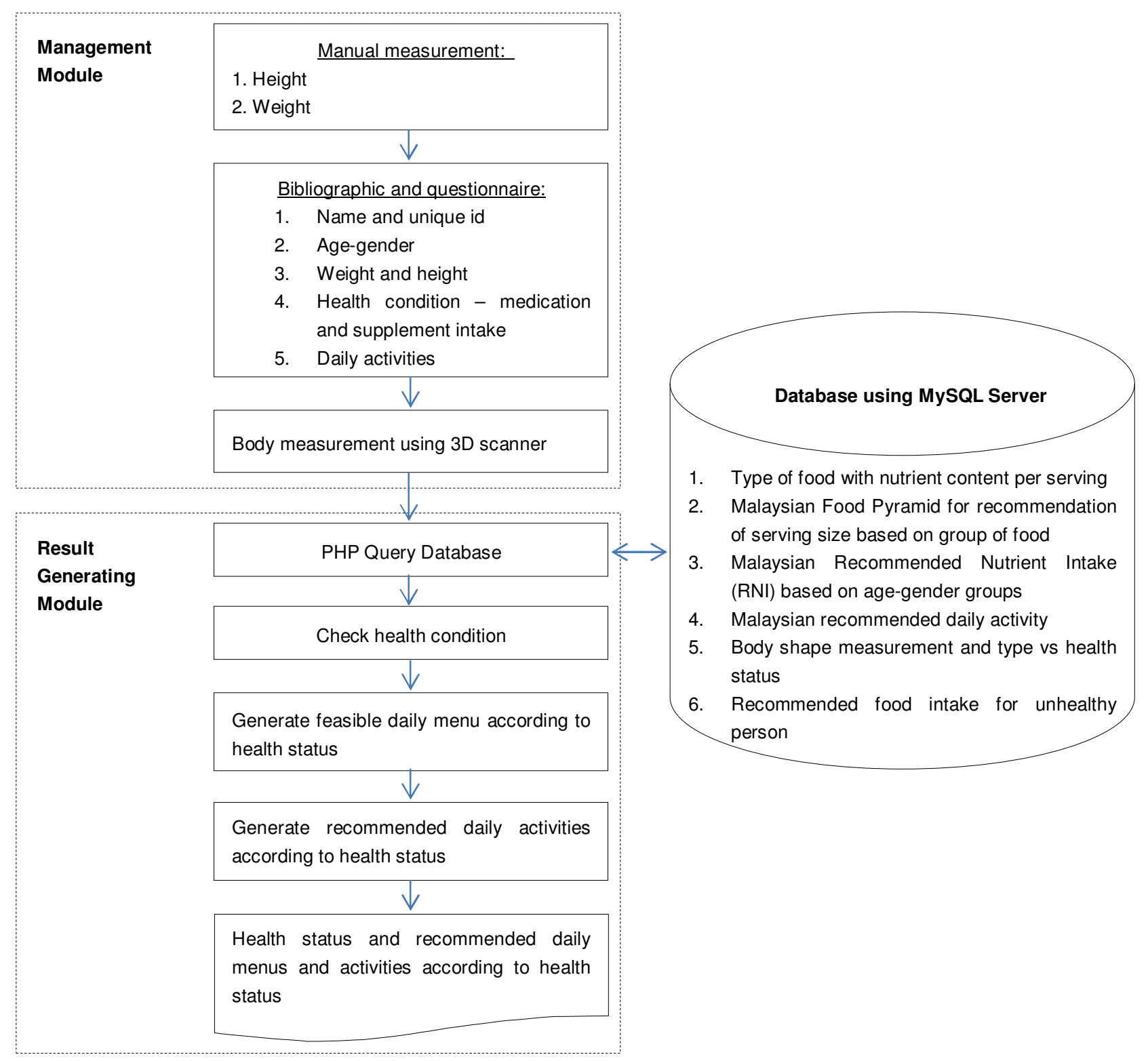

Fig. 2. The My_Healthy_Shape System Architecture

\section{Summary and Conclusion}

Our study hopes to propose a healthy daily diet and lifestyle for the general public. This study involves three phases which include the development of specialized databases, formulating suitable mathematical models and establishment of an interactive web-based educational-health guide system. The system offers a database of foods and drinks complete with the recommended serving sizes, which satisfy the Malaysian RNI. The system also provides recommended daily activities and health status of the users. Users can plan their daily dietary intake that satisfies their health status and lifestyle. Additionally, the system can help create awareness among the users on energy requirements and optimal diet. 


\section{References}

[1] T. Brandt and J. Yong, "Malaysian-German Chamber of Commerce \& Industry, Market Watch 2009 - The Food industry. The $10^{\text {th }}$ Malaysian International Food \& Beverage Trade fair. http://malaysia.ahk.de/fileadmin/user upload/Dokumente/Sektorreports/Market Waych 2009/Foo d E 09.pdf., accessed 2010.

[2] G L Khor, M S Zalilah, Y Y Phan, M Ang, B Maznah, A K Norimah, Perceptions of body image among Malaysian male and female adolescents Singapore medical journal (Impact Factor: 0.63). 04/2009; 50(3):303-11.

[3] Lee-Min Wong1 and Yee-How Say ${ }^{*}$ Gender Differences in Body Image Perception among Northern Malaysian Tertiary Students British Journal of Medicine and Medical Research, ISSN: 2231-0614,Vol.: 3, Issue.: 3 (July-September)

[4] 2014 Nexus Strategic Partnerships Limited, "Current health issues and progress in Malaysia." http://www.commonwealthhealth.org/asia/malaysia/current health issues and progress in mala ysia/, accessed 25 August 2014.

[5] A. M. Anderson and M.B. Earle, "Diet planning in the third world by linear programming and goal programming", in Journal of Operational Research Society, Vol.34, pp.9-16, 1983. 